\title{
La conceptualización de la vida social escolar
}

\author{
ALFREDO GOÑI \\ Universidad del País Vasco

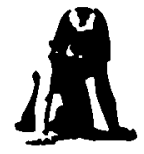

Resumen

El objetivo de este estudio era indagar a qué tipos de conceptos recurren los preadolescentes cuando razonan sobre sucesos ordinarios de la vida social escolar. Brinda trasfondo teórico a la investigación la hipótesis de los dominios de juicio social formulada por Elliot Turiel. Se mantuvo una entrevista de inspiración clínica con 96 alumnos de entre 10 y 16 años, distribuidos en tres grupos de edad. Como estimulos se presentaron cinco episodios representados en tiras de comics. El análisis de los resultados, más cualitativo que cuantitativo, permite detectar la complejidad conceptual de los episodios de la vida ordinaria y la pluralidad de categorias que conforman el conocimiento social; se clarifica también la entidad epistemológica de algunas nociones sociales a la par que algunos cambios de pensamiento asociados con la edad. Se apuntan, por fin, aquellas claves teóricas cuya profundización permitirá acercarse más al sujeto pensante real.

Palabras clave: Conceptos sociales, Conocimiento social, Dominios de conocimiento, Psicología de la educación, Moralidad, Convención, Prudencia, Privacidad.

\section{The conceptualization of school social life}

\begin{abstract}
The study investigates what type of concepts preadolescents resort to when they reason about ordinary events of their school social life. Elliot Turiel's theory on domains of knowledge offers a theoretical background to the investigation. An interview with a clinical orientation was passed to 96 students between 10 and 16 years of age arranged in three age groups. As a stimulus, five episodes were presented shown in comics strips. The analysis, more qualitative than quantitative, allowed us to detect the conceptual complexity of ordinary life episodes and the plurality of categories which make up social cognition. The data also clarified the epistemologic entity of some social notions as well as the thinking changes associated to age. Finally, those theoretical keys whose in depth study will allow to approach the real thinking individual are put forth.
\end{abstract}

Key words: Social concepts, Social cognition, Domains of knowledge, Educational psychology, Morality, Convention, Prudence, Privacity.

Dirección del autor: Universidad del País Vasco. E.U. del Profesorado de EGB de Alava. C/ Marqués de Urquijo s/n. 010006 Vitoria-Gasteiz.

Original recibido: Noviembre, 1988. Revisión recibida: Junio 1989. Aceptado: Junio 1989. 


\section{INTRODUCCION}

El enfoque cognitivo-evolutivo del desarrollo social (Baldwin, 1906); Piaget, 1932, G.H. Mead, 1934, Kohlberg, 1966, 1968, 1969) se esfuerza por explicar cómo los individuos, a partir de sus interacciones con el medio, van construyendo el conocimiento social y, más en concreto, cómo van formando sistemas conceptuales, progresivamente más elaborados, para interpretar los variados elementos de sus experiencias con los otros.

Elliot Turiel $(1979,1983)$, que comparte estos presupuestos, denuncia sin embargo que la mayoría de los estudios sobre desarrollo socio-cognitivo no parten de una clasificación sistemática de los dominios sociales; predomina, a su juicio, la idea de que el pensamiento humano sobre relaciones e instituciones se estructura primariamente en torno a nociones morales cuando es cabal dudar de que la estructura de la noción de justicia resulte lo suficientemente amplia como para organizar todo el conjunto de interacciones humanas incluidas las referentes a la intimidad, la atracción, el sexo o las formas de saludarse (Damon, 1977).

Sostiene Turiel que los juicios sociales del individuo no forman un sistema general unificado sino que la coherencia de las teorías sobre el mundo que los sujetos van construyendo, a medida que ordenan y transforman acontecimientos y sucesos, está ceñida a dominios específicos. Y presenta como hipótesis de trabajo un modelo del desarrollo socio-cognitivo en el que se diferencian tres dominios distintos del conocimiento social: el conocimiento moral (referente a las formas intrínsecas y obligatorias de organización de las interacciones entre sujetos); el conocimiento societal (de los sistemas sociales o de la matriz de las instituciones socio-culturales; y el conocimiento personal (sobre personas y sistemas psicológicos). Estos dominios de conocimiento experimentarían secuencias paralelas de desarrollo conceptual que pueden progresar a ritmos similares o desiguales.

La mayor parte del trabajo experimental de Turiel se ha dedicado a demostrar que la comprensión de la sociedad (lo societal) constituye un dominio de juicio diferente al moral y, más en concreto, que la diferenciación entre moralidad y convención emerge muy pronto en la vida, se mantiene durante la infancia y adolescencia y se prolonga a lo largo de la edad adulta. Pero al socaire de su hipótesis se ha generado un fecundo trabajo de exploración sobre cómo se construye el conocimiento social.

En consecuencia con la idea de que la naturaleza conceptual varía en función de cada tipo de interacción social, la heurística empleada por Turiel y colaboradores parte, en cada investigación, de una cuidadosa selección de acontecimientos-estímulo de la vida social. Turiel $(1975,1979,1983)$, Nucci y Turiel (1978), Nucci (1981), Nucci y Nucci (1982); Smetana $(1981,1983,1984,1986)$; Killen (1985); Laupa y Turiel (1986); Tisak y Turiel (1984); Tisak, (1986); Weston y Turiel (1980); Stoddart y Turiel (1985) han seleccionado sucesos de naturaleza prototípica de un dominio de juicio y persiguen describir el modo de razonar que elicitan y la progresiva reestructuración de cada tipo 
de conceptos. Así se va logrando describir la evolución en la comprensión de nociones tales como las de moralidad y convención y, en menor medida, las de prudencia y privacidad. Ello permite conocer la trayectoria evolutiva que experimenta el razonamiento sobre aquellos acontecimientos-tipo de la vida social cuya naturaleza se ajusta plenamente a alguna de estas categorías básicas del conocimiento social.

Pero sucede que los aspectos de la vida social son muy variados y que su naturaleza no es simple. La vida escolar, por ejemplo, se presenta como una realidad social compleja y poliédrica, constituida por acontecimientos de naturaleza persumiblemente variada y multidimensional. A nosotros nos interesó (Goñi, 1988) comprobar el grado de aplicabilidad de la teoría de los dominios de juicio al análisis del pensamiento sobre situaciones sociales ordinarias, esperando detectar aquellas limitaciones o carencias, tanto teóricas como empíricas, que precisen de mayor investigación.

Aun cuando no se trata directamente de delinear los cambios evolutivos que experimenta la comprensión de una u otra de las nociones sociales, el análisis de los modos de recurrir a distintas categorías conceptuales aportará probablemente nueva información sobre la naturaleza y entidad epistemológica de algunas nociones (lo socio-organizativo, lo prudencial, lo personal...) cuya provisionalidad es admitida desde la propia hipótesis de trabajo de Turiel.

De otro lado, poco se sabe acerca de las coincidencias y discrepancias al conceptualizar hechos sociales: en qué medida cumple un concepto la condición de referencia exclusiva y suficiente o tan sólo de referencia necesaria y principal para expresar la naturaleza de un suceso social; si en ocasiones se recurre a más de un tipo de nociones para dar cuenta de la naturaleza multidimensional de las conductas... Las investigaciones previas se han interesado más por los cambios evolutivos en las concepciones sociales que por analizar la coexistencia en un mismo sujeto de diversos tipos de juicios sociales y su uso, coordinado o no.

\section{METODO}

\section{Sujetos}

Participaron en la investigación 96 alumnos de edades comprendidas entre 10 y 16 años divididos en tres grupos de edad, cada uno de 32 sujetos, que corresponden a tres niveles educativos: $A$ ) 5.9 curso de EGB con una media de edad de 10 años y 10 meses; B) 8.9 de EGB cuya edad promedio era de 13 años y 10 meses; C) $10 .{ }^{\circ}$ curso (2.9 Enseñanzas Medias), con una media de 16 años y 3 meses.

Todos asisten a colegios de Vitoria-Gasteiz ubicados en las distintas zonas, céntricas y periféricas, de la ciudad. La mitad de los alumnos van a centros privados y la otra mitad a colegios públicos. 


\section{Procedimiento}

Con cada alumno se mantuvo una entrevista de una hora aproximada de duración, de la que aquí no se comenta más que una parte. Toda la conversación se grababa en magnetófonos y posteriormente se transcribía literalmente. Se contó con un recurso técnico no muy habitual, si bien utilizado por Nucci (1981), consistente en la utilización de viñetas o comics. El empleo de esta técnica se mostró útil para captar el interés, ahorrar digresiones verbales y fijar la atención en los temas de análisis.

En las tiras de comics aparecen representados cinco episodios escolares de naturaleza diversa, que pretenden ser significativos del amplio espectro de situaciones sociales escolares. El entrevistador proporciona una interpretación cerrada que guía los sucesivos pasos de la exploración: los protagonistas de las historias se comportan así de forma voluntaria y consciente, con cierta asiduidad, sin que concurran circunstancias excepcionales como estar muy enfadados, y sin mediar urgencias $\mathfrak{u}$ otros imponderables.

Los episodios son los siguientes: 1. Mikel, por las buenas, empuja y tira a Koldo, quien involuntariamente le está obstruyendo el paso; 2. Miren llega tarde al colegio; 3. Javi utiliza una ventana para salir del colegio; 4. Arantza no anota la fecha de un próximo control que el/la profesor/a acaba de anunciar; 5. Iñaki y Marian, dos hermanos, van al colegio vestidos a lo punki.

Las cuestiones, en su formulación más concisa, eran las siguientes:

1. ¿Qué te parece lo que bace el protagonista de esta bistorieta (Mikel, Miren...)?

2. ¿Por qué te parece (bien, mal, indiferente...) lo que bace?

Se prepararon preguntas alternativas para los casos en que los alumnos no explicitasen suficientemente su pensamiento.

\section{Códigos de baremación de respuestas}

A diferencia de otras líneas de investigación sociocognitiva, de las que el representante más significativo sería Kohlberg, en los trabajos de Turiel y colaboradores no se suele recurrir a una única codificación tipificada. Dado que cada investigación utiliza acontecimientos-estímulo propios se requiere elaborar códigos que se adecuen a las tareas utilizadas y a los objetivos previstos.

El análisis cualitativo de los argumentos, a partir de un reducido primer grupo de transcripciones, nos llevó a distribuirlos en cinco categorías conceptuales (Cf. Apéndice), que se utilizarán como variables experimentales, y que se entienden provisionalmente del modo siguiente:

A) Argumentos de naturaleza moral. Que tienen que ver con aquellas acciones en las que intrínseca y directamente se atenta contra el bienestar y derechos de los demás, es decir, con cuestiones de justicia. 
B) Argumentos de indole societal. En los que se apela a necesidades de la organización social o de mantener uniformidades conductuales (convenciones) que coordinan las interacciones de los individuos dentro de los sistemas sociales. Turiel, del que tomamos prestado el concepto, entiende que al razonar tanto sobre los aspectos socio-organizativos como sobre los socio-convencionales se pone de relieve una misma estructura conceptual subyacente, el conocimiento societal, el relativo a la organización social. Revisaremos, a la luz de los resultados, si se puede dar por buena tal afinidad o si, por el contrario, sería preferible entenderlos como áreas conceptuales distintas.

C) La categoría de evitación-autoridad. Agrupa argumentos de justificación en los que los sujetos apelan a la importancia de las normas establecidas o al castigo que la autoridad, o los semejantes, pueden infringir a quienes realicen una determinada acción.

D) La noción de lo prudencial. Se aplica a acciones que intrínseca y directamente interesan al bienestar físico o psicológico del agente, aun cuando indirectamente puedan afectar a otros. Las consecuencias negativas para uno mismo (o para los demás), a diferencia de los castigos, no son impartidas por agentes sociales.

E) Mediante conceptos personales. El sujeto apela al derecho a una privacidad de decisión en función de sus gustos y preferencias. Tales conceptos se elicitan ante situaciones en las que ni se atenta contra el bienestar o derechos de otros ni se afecta a un tú genérico; dependen por tanto tan sólo de las preferencias y derechos individuales.

\section{RESULTADOS}

Comentaremos por separado la valoración que merece en cada episodio el proceder del protagonista y, en segundo lugar, la justificación de esa valoración.

\section{Juicios sobre los cinco sucesos}

Tres tipos de valoraciones se emiten (Cf. Tabla 1) ante las conductas en cuestión; a las esperables categorías de Bien y Mal se añade la de Indefinición.

Se incluyen en la categoría Bien aquellas respuestas que consideran o valoran el acto positivamente. Constituyen la categoría $\mathrm{Mal}$ los juicios que consideran o valoran el acto negativamente. Se reserva la codificación de Indefinición para aquellas respuestas indefinidas o ambiguas en las que no hay una valoración única y clara, ni positiva ni negativa, del acto: se afirma que en parte está bien y en parte mal, se 


\section{6}

condiciona el juicio del acto a las posibles consecuencias que de él se deriven...

\section{TABLA I}

Porcentajes en el modo de juzgar cinco sucesos escolares

\begin{tabular}{lrcc}
\hline & Mal & Bien & Indefinición \\
\hline Suc. 1. & 100 & - & - \\
Suc. 2 & 100 & - & - \\
Suc. 3 & 98 & - & 2 \\
Suc. 4 & 72 & 21 & 7 \\
Suc. 5 & 21 & 40 & 39 \\
\hline
\end{tabular}

Una primera aproximación al enjuiciamiento de los sucesos, a partir de la valoración del acto, pone de relieve la clara diferencia establecida entre los tres primeros y los dos últimos episodios:

- Las tres primeras acciones (empujar, llegar tarde, salir del colegio por la ventana) reciben una valoración negativa.

- Sin embargo, mientras para el $72 \%$ de los sujetos está mal el no anotar la fecha de un examen (episodio 4), a un $21 \%$ le parece bien. El $7 \%$ restante hace depender la bondad o malicia de la acción de la actitud del alumno ya que, en su opinión, no hay por qué asociarla necesariamente con desinterés.

- Sólo una minoría (21\%) considera que está mal la conducta de ir al colegio vestido a lo punki; por contra para un $40 \%$ está bien y el $39 \%$ restante lo ve como una acción ambigua con aspectos al mismo tiempo positivos y negativos.

\section{Los argumentos}

Se preguntaba: ¿Por qué te parece que está bien/mal/regular que el protagonista de esta historia actúe asi?

Empecemos por contabilizar el total de veces que se apela a consideraciones de una u otra índole al justificar por qué está mal, bien, o en parte bien y mal... cada una de las acciones (ver Tabla II).

\section{TABLA II}

Distribución de los distintos argumentos por sucesos

\begin{tabular}{ccccccc}
\hline & A & B & C & D & E & Total \\
\hline Suc. 1 & 123 & 13 & 8 & - & - & 144 \\
Suc. 2 & - & 45 & 35 & 56 & - & 136 \\
Suc. 3 & - & 72 & 27 & 48 & - & 147 \\
Suc. 4 & 1 & 20 & 6 & 80 & 4 & 111 \\
Suc. 5 & - & 56 & 1 & - & 76 & 133 \\
Total & 124 & 206 & 78 & 184 & 80 & 671 \\
\%. & 18 & 31 & 12 & 27 & 12 & \\
\hline \multicolumn{7}{c}{ A= Moral; B= Societal; C= Evitación; D= Prudencial; E= Personal. }
\end{tabular}


La mitad de las argumentaciones utilizadas en el razonamiento social no son ni de índole moral ni de índole societal. Han aflorado planteamientos de corte prudencial en número importante $(27 \%) \mathrm{y}$, en porcentaje menor, argumentos de evitación-autoridad (12\%) y personajes $(12 \%)$. En la medida en que los cinco sucesos seleccionados sean representativos del conjunto de la vida social contamos con una aproximación a las dimensiones cuantitativas del uso de las distintas categorías argumentales.

Sólo la acción de empujar y tirar a alguien (suceso 1) es considerable de forma mayoritaria como asunto de índole moral mientras que a los demás sucesos no se les atribuye consideraciones morales. Contrasta, además, la considerable concordancia en admitir la naturaleza moral de tal acción frente a la amplia divergencia en la selección de una única categoría de juicio que dé cuenta de la naturaleza de los sucesos no-morales.

Una presentación diferente de los mismos datos permite observar, en la Tabla III, el porcentaje de sujetos que o bien apelan a una sola argumentación al conceptualizar cada suceso o bien recurren a una conceptualización plural o múltiple porque lo ven polifacético....

TABLA III

Porcentaje de sujetos que recurre a un solo o más de un tipo de argumento

\begin{tabular}{llrrrrr}
\hline & A & B & C & D & E & Múltiple \\
\hline Suc. 1 & 79 & - & 1 & - & - & 20 \\
Suc. 2 & - & 21 & 14 & 28 & - & 37 \\
Suc. 3 & - & 40 & 10 & 9 & - & 41 \\
Suc. 4 & - & 9 & - & 74 & 2 & 15 \\
Suc. 5 & - & 20 & 1 & - & 41 & 3 \\
\hline
\end{tabular}

$\mathrm{A}=$ Moral; $\mathrm{B}=$ Societal; $\mathrm{C}=$ Evitación; $\mathrm{D}=$ Prudencial; $\mathrm{E}=$ Personal.

Queda en evidencia la naturaleza conceptual compleja de los sucesos sociales, aun de los aparentemente sencillos; lo habitual no es toparse con sucesos prototípicos de un solo dominio de juicio sino con situaciones que admiten perspectivas complementarias y que, en casos, resultan ambiguos por lo que surgen discrepancias acerca de su naturaleza. Veamos, en concreto, cómo se conceptualiza cada uno de los episodios:

- Todos ven mal que se empuje y tire y todos lo condenan por tratarse de una acción que atenta directamente contra el bienestar físico o psicológico de otra persona o contra sus derechos. Se trata de un asunto prototípicamente moral, sólo percibido por un sujeto (que apela a la ley de Dios para considerarlo como malo) de forma discrepante. El $79 \%$ recurre exclusivamente a ese razonamiento mientras que algunos ( 13 alumnos, sin que se detecten diferencias de edad), complementan los argumentos morales con otros de índole societal («pegar a alguien incitará a grescas y peleas» y «es de mala educación») o de evitación del castigo («se ganará mala fama»). 
- Se discrepa en el modo de conceptualizar la impuntualidad. Un $28 \%$ coincide en valorarla como un asunto de naturaleza exclusivamente prudencial; pero un $30 \%$ añade otras consideraciones (organizativas, de evitación...) a la dimensión prudencial, y el resto (42\%) discrepa de que sea asunto prudencial.

- La acción de salir del colegio por la ventana en lugar de por la puerta es contemplada exclusivamente desde perspectivas societales en una u otra de sus versiones, la socio-organizativa y/o socio-convencional, por un $40 \%$ de los alumnos; un $32 \%$ añade a lo societal consideraciones de tipo prudencial o de evitación.

Discrepa, sin embargo, de que la naturaleza de este suceso sea societal un $28 \%$, concibiéndolo meramente como una cuestión de prudencia, según una desigual distribución por edades: $15 \%, 8 \%$ y $5 \%$. Esta diferencia es estadísticamente significativa (Chi Cuadrado 6,49 a un n.c. 5\%) y pone de relieve la tendencia de los más pequeños a percibir dimensiones prudenciales donde los mayores ven aspectos de organización social.

- El no anotar la fecha de un examen es contemplado mayoritariamente en relación con el aprovechamiento académico (suceso 4) y, en consecuencia, se destaca el componente prudencial de la acción al establecer una conexión entre esa tarea escolar y el logro del éxito académico: en caso de no anotar no se acordarán de la fecha del examen, no aprenderán o suspenderán. Los menos ( 6 sujetos) entienden que, si no anotan, se les castigará. Algunos (15\%) complementan los argumentos prudenciales con razones socio-organizativas: el anotar evita problemas de organización escolar o la actitud correcta de un estudiante es ir anotando las cosas; son pocos $(9 \%)$ los que lo argumentan tan sólo desde esa perspectiva societal.

Parece especialmente relevante en esta situación tanto el hecho de que el $71 \%$ de los alumnos interprete el acto en un contexto de aprendizaje como el que, sin embargo, el $29 \%$ restante niegue la relación medio-fin entre anotar o no y rendir en lo académico o no. El $29 \%$ de alumnos que establecen algún tipo de disociación entre el hecho de no anotar y los efectos negativos para el sujeto no se distribuye homogéneamente en los tres grupos de edad: 3 son de $5 .^{\circ}, 12$ de $8 . .9 \mathrm{y}$ 14 de $10 .$. curso, lo que resulta una diferencia asociada con la edad estadísticamente significativa (Chi Cuadrado 7,10) a un n.c. del 5\%. La relevancia de este dato reside en que el componente prudencial de las situaciones parece que tiende a ser reinterpretado de forma nueva con la edad: las consecuencias negativas no siempre son automáticas e incluso, aunque lo fueran, es asunto propio de cada cual el correr con ellas.

El suceso 5 , el ir al colegio vestido a lo punki, se configura como una situación conflictiva y ambigua en cuanto que las discrepancias o las dudas de la totalidad de los sujetos se plantean entre dos alternativas de juicio: lo societal o lo personal. El $41 \%$ de los sujetos lo percibe como asunto personal, el $20 \%$ societal y el $39 \%$ restante considera tanto los aspectos personales como los societales del episodio.

Todos los sujetos que conceptualizan como asunto exclusivamente societal el ir al colegio a lo punki, lo valoran categóricamente mal por quebrantar expectativas convencionales (aunque en algunos casos se 
alude también a repercusiones organizativas negativas): se trata de 15 alumnos de 5.9; de 2 de $8 .^{\circ}$ y de otros 2 de 10.. . A parece con claridad (Chi Cuadrado 15,77) una evolución asociada con la edad.

De otro lado, todos los sujetos a los que les parece bien tal proceder $(40 \%)$ conceptúan la acción como una cuestión exclusivamente personal; algunos incluso argumentan que estaría mal renunciar al derecho a vestir como se quiera por más que su uso puede acarrear consecuencias negativas. Ese $40 \%$ de sujetos se reparte en un $7 \%$ de alumnos de 5.9 , en un $12 \%$ de $8 .{ }^{\circ}$, y en un $21 \%$ de $10 .{ }^{\circ}$ curso (Chi Cuadrado 7,55 significativo a un n.c. $5 \%$ ).

Se dan, por tanto, tres tipos de valoraciones del episodio de ir al colegio a lo punki: a) El grupo de alumnos de más edad, en su mayoría ( $65 \%$ ) lo ven como asunto personal y de libre elección; b) Los más jóvenes tienden a considerarlo de forma negativa por quebrantar exigencias convencionales. Así se manifiestan la mitad de los alumnos de 5.․ además otro $28 \%$ de ese grupo de edad no acierta a estructurar argumentos a favor y en contra; c) Lo que caracteriza fundamentalmente a los de 8.9 es que ocupan una posición intermedia: la mitad de ellos optan por lo convencional o por lo prudencial y la otra mitad pondera tanto consideraciones societales como personales, sin resolver la contradicción de ambos componentes de la situación.

Destaquemos que de los 56 sujetos que o bien sólo contemplan la dimensión personal, o entienden que lo personal prevalece sobre consideraciones societales, 10 alumnos son de $5.9,16$ de 8.9 y 30 de enseñanzas medias, lo cual supone diferencias estadísticamente significativas (Chi Cuadrado 11,29) a un n.c. del $1 \%$. Se aprecia un cambio evolutivo en la conceptualización de una situación conflictiva convencional-personal al producirse un progresivo mayor aprecio por los valores personales.

De este conjunto de información pueden extraerse algunas conclusiones:

1. En el análisis del razonamiento social es preciso, a la par que operativo y fecundo, el distinguir varias categorías conceptuales: el conocimiento social se organiza y estructura en referencia a una pluralidad de nociones básicas. Una parte muy importante de las situaciones e interacciones sociales tiene que ver con el bienestar y/o derechos de los demás y con la comprensión de la organización social, por lo que las nociones morales y societales son núcleos primarios en torno a los que se estructura el conocimiento. Pero no son los únicos, otras categorías (la de evitación del castigo, la autoridad, la prudencia o la personal) organizan el razonamiento sobre sectores de la vida social cuya extensión es tan amplia como la cubierta por consideraciones morales o societales.

2. Caracteriza al razonamiento sobre sucesos ordinarios de la vida social el recurso combinado a juicios de diversas categorías conceptuales. Los sucesos ordinarios de la vida social son complejos, no siempre son enjuiciados de forma coincidente por todos los sujetos a partir de una única categoría conceptual, y con frecuencia requieren recurrir a más de un tipo de nociones sociales, sin que siempre se logre coordinarlas. 
Se vislumbran algunos criterios cuya depuración posibilitaría determinar el grado mayor o menor de complejidad (naturaleza prototípica, aditiva, ambigua o conflictiva) de los distintos sucesos sociales en referencia a la univocidad o multidimensionalidad conceptual que tienden a elicitar:

a. Las concordancias mayoritarias en recurrir a una sola categoría conceptual tienen lugar al juzgar conductas que atentan contra el bienestar y derechos de los demás (asuntos morales) y al razonar sobre tareas de aprendizaje (de naturaleza prudencial);

b. Por contra, las situaciones de naturaleza no-moral tienden a elicitar una amplia dispersión de juicios. Su naturaleza resulta compleja y ambigua por lo que se apela a consideraciones de más de un tipo (prudenciales, socio-organizativas, convencionales, de evitación...) y abundan las discrepancias;

c. Carácter especialmente dilemático y conflictivo presentan algunos episodios cuya conceptualización requiere la opción entre/la coordinación de dos dimensiones contrapuestas. Así se han mostrado los sucesos sociales que, si bien pueden estar regulados por convenciones o reclamar una reflexión prudencial, al mismo tiempo pudieran ser entendidos como asunto de libre decisión personal.

3. En la conceptualización de sucesos ordinarios se han ido señalando diferencias asociadas con la edad, de las que merecen destacarse dos:

a. Se constatan discrepancias interindividuales al considerar el rango prudencial o societal de algunas conductas y la naturaleza prudencial o personal de otras; en tales casos los pequeños tienden a fijarse en el componente prudencial de las situaciones mientras que los mayores prestan atención a lo no-prudencial;

b. Cuando resulta conflictivo decidir entre la dimensión convencional o de privacidad personal de una situación, los pequeños tienden a subrayar lo convencional mientras que los mayores dan principal importancia a lo personal; los medianos muestran dificultad en decidirse por una u otra dimensión.

\section{DISCUSION}

Categorías conceptuales y dominios de juicio

No es momento para entrar a discutir si goza de suficiente solidez científica la tesis de que los dominios de juicio social conforman estructuras parciales del conocimiento (Goñi, 1989a). En cualquier caso, y con cierta autonomía de sus hipótesis teóricas, la más valiosa aportación de Turiel ha consistido en impulsar una nueva vía de análisis del conocimiento social, una heurística de investigación, en la que son las nociones o categorías conceptuales, y no directamente los dominios, las variables de trabajo experimental. $\mathrm{Ha}$ de reconocerse que, por el momento, los datos sobre la construcción de nociones sociales son insuficientes y provisionales, con lo que resulta prematuro el empeño de atar todos los cabos de una nueva teoría del conocimiento social; 
pero, al mismo tiempo, quedan abiertos un campo y una vía de trabajo donde no cabe otra estrategia que la propuesta por Turiel de establecer procesos de ida y vuelta entre definiciones provisionales y confrontación con los resultados empíricos de la investigación.

Veamos en qué medida nuestros datos afectan a lo concluido a partir de investigaciones previas:

1. Al estudiar el desarrollo del juicio moral ha urgido destacar los aspectos evolutivos y secuenciales, la reestructuración evolutiva de la noción de justicia y el tránsito de una moralidad heterónoma a otra autónoma, más que los elementos constantes y comunes en ambos estadios. Pero, el alcanzar una moralidad autónoma no significa la extinción de las motivaciones heterónomas; tal supuesto conduciría a una imagen no sólo incompleta del desarrollo social sino incluso a una visión esquemática y muy alejada del sujeto pensante real. Piaget ya apuntó, aun sin desarrollar la cuestión (1932), que la moralidad autónoma ha de entenderse como un modo de razonamiento prioritario sobre las consideraciones heterónomas, que sin embargo no desaparecen.

Podemos afirmar, en efecto, que en el sujeto coexiste el razonamiento moral con otros tipos de juicios sociales; incluso se comprueba la tendencia, más acusada en los mayores, a complementar con perspectivas sociales su análisis moral de los comportamientos; a un pensamiento moral autónomo no le son ajenas, por ejemplo, consideraciones heterónomas de evitación-autoridad. Convendría profundizar en cómo coordina la persona moral autónoma su fidelidad a los principios de justicia con la salvaguarda de sus intereses personales y cómo delimita los ámbitos conductuales regidos por criterios de moralidad de aquéllos que no lo están.

2. Mostrábamos con anterioridad ciertas dudas sobre la coherencia interna de la noción de dominio de conocimiento societal, que abarcase al mismo tiempo la comprensión de la organización social y de las convenciones sociales. De un lado, es evidente que lo socio-organizativo y lo convencional no son sino dos aspectos de la organización social; ahora bien, los datos empíricos ratifican la existencia de suficientes diferencias como para desaconsejar el entenderlos como dos subcategorías de un mismo dominio de juicio:

- Unos y otros argumentos se aplican a sucesos diferentes. Así todas las razones societales alegadas en el suceso de llegar tarde al colegio y en el episodio de no-anotar la fecha del examen son socioorganizativas: «se perjudica el ritmo de la clase», «es un desorden», «así no se puede funcionar». Sin embargo, en el episodio de salir por la ventana (suceso 3 ) hay más razones de índole convencional que socio-organizativas: «se da una mala imagen del colegio», «es una gamberrada», «es salirse de lo establecido», «se quiere llamar la atención»...).

- No experimentan trayectorias evolutivas equiparables y siméticas. Lo socio-organizativo tiende a ser más valorado con la edad, por ejemplo, frente a lo prudencial; por el contrario, puede comprobarse con la edad una progresiva relativización de lo convencional.

- Parece característico de lo socio-organizativo el darse entreverado con otras dimensiones y, en especial, con las prudenciales. Por 
contra, las consideraciones convencionales terminan por entrar en cierto conflicto con el ámbito de decisión personal.

3. La conceptualización de lo prudencial requiere expresa atención en el estudio del conocimiento social ya que son muchas las situaciones en las que puede producirse, sin mediación social, un perjuicio directo para uno mismo o indirecto para los demás. Baste recordar que la conceptualización básica de lo que en otros estudios se ha identificado como "procesos o tareas de aprendizaje» tiene que ver directamente, según nuestros datos, con el modo de entender lo prudencial y no tanto con la comprensión de la organización social (DodsworthRugani, 1982).

Apelar a razones de prudencia no debe quedar asociado a un tipo de moralidad heterónoma y regida por criterios egoístas; tiene una entidad propia desde la primera infancia y en la edad adulta. En cualquier caso proponemos que se distingan dos subcategorías en la prudencia: a., lo que afecta negativamente a la integridad física; $b$., lo que afecta negativamente al autodesarrollo (aprendizaje, aprovechamiento escolar...).

4. La comprensión de uno mismo como alguien con una esfera privada de decisión personal se va asentando en la preadolescencia, si no antes. Hemos contestado que sucesos que en una edad inferior tienden a percibirse en su vertiente convencional o prudencial, en edades superiores se inscriben en un marco de consideraciones personales. $\mathrm{La}$ conceptualización personal de un suceso no parece requerir la negación de aspectos previos y básicos, como lo convencional o lo prudencial, sino que consiste en una nueva percepción que asume la anterior. El sujeto, a partir de un momento de su edad, empieza a comprender que la regulación convencional de algunas conductas no requiere obligado cumplimiento y que lo prudencial de por sí no es la razón última de la toma de decisiones porque tal vez no todo lo que a uno perjudica haya que dejar de hacerlo.

A expensas de nueva información, proponemos entender lo personal como ese ámbito de privacidad que el individuo va progresivamente ganando y liberando de la normativización socio-convencional o prudencial. El sujeto va constituyendo evolutivamente una esfera de privacidad arrancándola de parcelas que previamente han estado arbitradas por convenciones o por la referencia prudencial.

\section{El razonamiento sobre la realidad escolar}

Esta vía de análisis del razonamiento social, ¿permite avanzar en la comprensión de cómo razonan los alumnos sobre las interacciones sociales que conforman la vida escolar? La primera comprobación es que la aplicabilidad de la teoría de los dominios de juicio al análisis del razonamiento sobre sucesos habituales de la vida social falla si se espera disponer de una especie de sistema clasificatorio según el cual a cada suceso social habría de corresponder una única categoría conceptual.

Tratar de trasladar directamente los resultados de la investigación 


\section{3}

a las situaciones concretas de razonamiento sería ceder a tentaciones psicologizantes y extrapolativas. Detectar la existencia plural de juicios sociales no conlleva el efecto de disponer de un instrumental de aplicación inmediata al razonamiento sobre sucesos escolares, ya que la mayoría de ellos vienen caracterizados por su naturaleza multidimensional y se necesita seguir clarificando, en especial, la combinación de conceptos en el juicio de sucesos ordinarios (Goñi, 1989b).

Si hubiésemos de proponer cuáles son las claves a las que hay que prestar atención al evaluar el razonamiento social de los alumnos indicaríamos tres: A) Analizar previamente el contenido de la tarea distinguiendo los posibles aspectos (morales, socio-organizativos, socioconvencionales, de evitación-autoridad, prudenciales o personales) que el tema objeto de reflexión pueda encerrar; B) Atender al grado mayor o menor de complejidad formal que pueda requerir según se prevea que la naturaleza del asunto sea prototípica de un campo conceptual, o admita más de una perspectiva de análisis, o tal vez resulte ambigua o conflictiva su definición; C) El nivel de competencia alcanzado por el sujeto en las diversas categorías conceptuales a las que recurre en cada caso.

\section{Apéndice}

\section{CATEGORIAS DE JUSTIFICACION DE LOS JUICIOS ACERCA DE LOS CINCO EPISODIOS}

\section{Descripción}

\section{Categorias}

A. Argumentos de naturaleza moral que apelan al bienestar, integridad física o derechos de otras personas diferentes a la que realiza el acto.

A.1. Referencia a los efectos intrínsecos negativos para el bienestar físico de otros ( «le hace daño» o «le puede hacer una herida»; «se puede caer encima de alguien»); para el bienestar psicológico de otros («le humilla», «le atemoriza», «le pica», «le hace sentirse rechazado», «es como un insulto», «le chulea», «se cree superior a los demás»), o para el equilibrio o comodidad de otros («le tira todos los papeles», «perjudicas»).

A.2. Apelación a principios generales de justicia, reciprocidad y derechos de las personas. Se hace referencia al mantenimiento de un equilibrio de derechos entre las personas («tienen derecho los dos», «abusa», «es una desconsideración», «se debe ser amigos», "no es justo», «debió pedirle permiso», "no se puede ir así por la vida»); al compañerismo («así no se porta un amigo»); a la reciprocidad («a mí no me gusta que me traten asì»); al respeto, a la consideración personal 


\section{4}

o a los derechos de la persona («es su forma de ser», «tiene derecho a que le respeten y a que no le juzguen mal»).

B. Argumentos de índole societal. Apelación a la necesidad de organización social o de mantener un sistema de expectativas compartidas entre las personas.

B.1. Se hace referencia a las consecuencias negativas para aspectos funcionales del orden social («sería un desastre», «sería una desorganización», «se incitaría a la gente a no comportarse de forma civilizada», «se producen atascos», «interrumpes», «rompes el ritmo de la clase») o bien para aspectos estructurales del orden social, como el uso correcto de las cosas («para algo están las puertas») o para el sentido e imagen de instituciones sociales («a eso no se va al colegio», «se da una mala imagen del colegio», «le haces esperar», «desconcentras al profesor»).

B.2. Se hace referencia a desviaciones de convenciones o expectativas conductuales consideradas como correctas («es de mala educación», «está un poco loquillo», «es extraño», «lo hace por llamar la atención», «es propio de personas inmaduras», «es irresponsable», «debe comportarse como todos los demás», "parece que no tiene ganas de estudiar», "parecen subnormales»).

C. Orientación a la autoridad, normas y castigos.

C.1. Apelación a la aprobación de figuras específicas de autoridad («lo ha mandado la señorita») o a la existencia de reglas («está mandado», «hay una regla»).

C.2. Se alude a consecuencias o reacciones sociales negativas de evitación y castigo hacia el autor, incluyendo rechazo social («te echarán una bronca», «dejará de ser amigo», «te ponen mala fama», «te miran mal»).

D. Razones de prudencia. Se hace referencia a las consecuencias negativas, sin mediación social, para la persona que lo hace.

D.1. Se alude a los efectos negativos para el confort, bienestar o salud personal («te puedes hacer daño», «se puede romper una pierna», «se aburrirá»).

D.2. Referencias a efectos negativos para el autodesarrollo o realización de uno mismo («el saldrá perdiendo», «no aprenderá», «se le olvidará», «seguirá siendo tímido», «no se acostumbrará a ser puntual»).

E. Apelación a preferencias o prerrogativas personales.

E.1. Se alega el que se trata de un gusto, preferencia o elección inocua («no haces mal a nadie», «es su gusto», «es libre de hacerlo», «es su responsabilidad»).

E.2. Se subraya el carácter de elección prioritaria frente a lo prudencial («es su vida y puede hacerlo, aunque se haga daño..., le miren mal..., se rían de él) o el rechazo social («él tiene que decidir si prefiere una cosa u otra») o frente a derechos de otros: puede hacerlo aun cuando a otros no les guste y puedan resultar indirectamente perjudicados. 


\section{Referencias}

BALDWIN, J.M. (1906). Social and ethical interpretations in mental development: a study in social psychology, Nueva York: MacMillan, 1987.

DAMON, W. (1977). The social world of the child, San Francisco: Jossey-Bass.

DODSWORTH-RUGANI, K.J. (1982). The development of concepts of social structure and their relationships to school rules and authority, Tesis doctoral inédita, Berkeley: Universidad de California.

GoÑ, A. (1988). Razonamiento social sobre conductas escolares. Pluralidad y coordinación de categorias conceptuales, Edición en microficha. Servicio de Publicaciones: Universidad del País Vasco.

GoNI, A. (1989a). «Los dominios del conocimiento social: la hipótesis de Elliot Turiel (I)» y «Los dominios del conocimiento social: heurística y teoría (II)». Aula Abierta, 52, 31-83, ICCE de la Universidad de Oviedo.

GoÑı, A. (1989b). «La coordinación de conceptos en el razonamiento sobre situaciones sociales complejas». Boletín del ICCE de la Universidad Autónoma de Madrid, 14, 5-20.

KILLEN, M. (1985). Children's coordinations of moral, social and personal concepts». Tesis doctoral no publicada. California: Berkeley.

KoHlberG, L. (1966). «Moral education in the schools», en The School Review, 74, 1-30.

KoHlberg, L. (1968). «The child as moral philosopher». En Psychology Today, [Traducción: «El niño como filósofo moral», en Delval, J. (Comp.): Lecturas de psicología del niño. Vol. 2.: El desarrollo cognitivo $y$ afectivo del niño y del adolescente, 303-314, Madrid: Alianza, 1983 (3. ${ }^{a}$ ed.)]

KOHLBERG, L. (1969). «Stage and sequence: The cognitive-developmental approach to socialization», en GosLIN, D.A. (ed.): Handbook of socialization theory and research, Chicago: Rand McNally.

LAUPA, M.; TURIEl, E. (1986). «Children's conception of adult and peer authority». Child Development, 57, 405-412.

MEAD, G.H. (1934). Mind, self and society, Chicago: University of Chicago Press.

NUCCI, L.P.; TURIEL, E. (1978). "Social interactions and the development of social concepts in preschool children». Child Development, 49, 400-407.

NUCCl, L.P. (1981). «Conceptions of personal issues: a domain distinct from moral or societal concepts». Child Development, 52, 114-121.

NUCCI, L.P.; NUCCI, M.S. (1982). «Children's social interactions in the context of moral and conventional transgressions». Child Development, 53, 403-412.

PiageT, J. (1932). Le jugement moral chez l'enfant, Paris: Presses Universitaires de France. [Traducción: El criterio moral en el niño. Fontanella, Barcelona, 1971].

SmEtanA, J.G. (1981). «Preschool children's conceptions of moral and social rules». Child Development, 52, 1333-1336.

SmetanA, J.G. (1983). «Social-cognitive development: domain distintions and coordinations». Developmental Review, 3, 131-147.

SMETANA, J.G. (1984), «Toddler's social interactions regarding moral and conventional transgressions». En Child Development, 55, 1767-1776.

SmETANA, J.G. (1986). «Children's impressions of moral and conventional transgressors». Developmental Psychology, Vol. 21, núm. 4, 715-724.

STODDART, T.; TURIEL, E. (1985). «Children's concepts of cross-gender activities». Child Development, S6, 1241-1252.

TISAK, M.S.; TURIEL, E. (1984). «Children's conceptions of moral and prudencial rules». Child Development, 55, 1030-1039.

TiSAK, M.S. (1986). «Children's conceptions of parental authority». Child Development, 57, $166-176$

TURIEL, E. (1975). «The development of social concepts: mores, customs, and conventions». En DePalma, D.J.; Foley, J.M. (Eds.), Moral development: Current theory and research, 7-38, Hillsdale, New Jersey: Lawrence Erlbaum Associates.

TuRIEL, E. (1979). "Social convention and morality: Two distinct conceptual and development systems». En KEASEY, C.B. (Ed.), Nebraska Symposium on Motivation, Vol. 25, University of Nebraska Press: Lincoln.

TURIEL, E. (1983). The development of social knowledge, Nueva York: Cambridge University Press. [Traducción: El desarrollo del conocimiento social. Moralidad y convención, Madrid: Debate, 1984].

WESTON, D.R.; TURIEL, E. (1980). "Act-rule relations: children's concepts of social rules». Developmental Psychology, Vol. 16, núm. 5, 417-424. 


\section{6}

\section{Extended Summary}

This work introduces an investigation which tries to bring light to how five ordinary and representative episodes of social school life are conceptualized by preadolescents. The subject of study was based on Elliot Turiel's theory about the domains of social cognition. The heuristics of investigation used by Turiel and his collaborators are also useful as a reference. However, the interest of the research does not emphasize so much on outlining the developmental changes that the understanding of some or the other social notions undergo but the way in which they resort to some concepts or other when reasoning about standard events in ordinary life.

An interview with a clinical orientation was passed to 96 students of public and private schools in Vitoria-Gasteiz (Basque Country). The individuals form three age groups whose average age is 11,14 and 16 years. The were asked what they thought about five school behaviours. 1. Pushing; 2 . Arriving late to school; 3 . Leaving the school through a window; 4 . Not writing down the date of the next exam; 5. Going to school dressed as a punk rocker, and to give explanations for their answers. These episodes were presented orally as well as on comics strips.

The results reveal that social cognition of life is organised in relation to several conceptual categories, the tabbing of answers forced to open five categories of argumentation: moral, social, avoidance of punishment/authority, prudential and personal. It is also revealed that the mayority of events in every day life are complex and, therefore, their judgment demands the coordination of different kinds of reflexion. The main differences associated to the age in the conceptualization of school episodes is brougth about due to the fact that events which at a younger age tend to be seen in their conventional or prudential side, at an older age are inscribed in a frame of personal considerations.

Some aspects of.Turiel's theory are tinged with the new obtained information. Thus, too many differences are reported between the socio-organisational and the conventional facts which Turiel understands as subcategories of the social domain; the importance of the concepts related to the free sphere of personal decision are stressed and the theoretical keys in which research must go on are indicated in order to get a greater applicability of Turiel's theory to the analysis of reasoning about ordinary situations. 